\title{
Carcinosarcoma (malignant mixed mesodermal tumour) of the uterus: clinicoimmunohistochemical and histogenetic characteristics
}

\author{
Witold Kedzia $^{1,2}$, Dominik Pruski ${ }^{1,2}$, Kinga Iwaniec ${ }^{2}$, Marcin Przybylski ${ }^{1}$, \\ Zbigniew Friebe ${ }^{1}$, Helena Rajpert-Kedzia ${ }^{3}$
}

${ }^{1}$ Devision of Gynecology, Department of Perinatology and Gynecology, Karol Marcinkowski University of Medical Sciences, Poznan, Poland

${ }^{2}$ Laboratory of Cervical Pathophysiology, Gynaecology and Obstetrics Clinical Hospital, Karol

Marcinkowski University of Medical Sciences, Poznan, Poland

${ }^{3}$ Department of Pathomorphology, Gynaecology and Obstetrics Clinical Hospital, Karol

Marcinkowski University of Medical Sciences, Poznan, Poland

\begin{abstract}
To search for favourable prognostic factors in carcinosarcoma (CS) on the basis of clinical, morphological and immunocytochemical data, while simultaneously considering the histogenesis of this neoplasm. Thirty two uterine CS patients were analysed based on clinical and morphological data. In addition, each specimen was examined by immunohistochemistry with antibodies characteristic for relevant types of cells and tissues. The presence of both carcinomatous and sarcomatous patterns was observed in all tumours. Among carcinomatous patterns, endometrioid carcinoma was the commonest, while serous, clear cell, and undifferentiated carcinomas were less common. Among sarcomatous patterns, endometrioid sarcomas represented the largest group, while leiomyosarcomas, chondrosarcomas, fibrosarcomas, osteosarcomas, and rhabdosarcomas were rarely observed. Mitotic activity was evidently higher in carcinomas. In seven cases, the expression of both cytokeratin and vimentin was noted in cells of carcinomatous patterns. We found that an early diagnosis (stage I-II) and an initially aggressive surgical cytoreduction were favourable prognostic factors in CS. Furthermore, the presence of cytokeratin-vimentin positive cells in carcinomatous patterns suggests sarcomatous metaplasia of adenocarcinoma. However, the prognostic value of various histological structures of carcinosarcomas could not be identified. (Folia Histochemica et Cytobiologica 2012, Vol. 50, No. 4, 513-518)
\end{abstract}

Key words: uterine neoplasms, carcinosarcoma, immunocytochemistry, histological pattern, endometrioid carcinoma, prognosis

\section{Introduction}

Carcinosarcomas (CS), also known historically as malignant mixed mesodermal tumours, are considered to be one of the most aggressive uterine cancers

Correspondence address: D. Pruski, Laboratory of Cervical Pathophysiology, Gynaecology and Obstetrics Clinical Hospital, Karol Marcinkowski University of Medical Sciences; Polna St. 33, 60-535 Poznan, Poland;

e-mail: dominik.pruski@oxytop.pl
[1-3]. They are most commonly composed of carcinomatous components and malignant mesenchymal components. Because of their complicated histogenesis, there are numerous uterine carcinosarcoma classification hypotheses, and debate continues as to whether CS is monoclonal or biclonal. However, most authors agree with the 'combination hypothesis', which states that CS originates from high-grade carcinoma with sarcomatous differentiation. The 'conversion hypothesis' states that CS is derived from a single stem cell population which is multipotent or has a potential for metaplastic transformation. On the 
Table 1. Primary antibodies and their respective immunophenotypes

\begin{tabular}{|l|c|c|c|}
\hline Antibodies* & Form & Dilution & Immunophenotype \\
\hline CD10 & Monoclonal & $1: 80$ & Endometrial stromal sarcoma \\
\hline CD117** & Polyclonal & $1: 500$ & Tyrosine kinase transmembrane receptor \\
\hline Vimentin & Monoclonal & $1: 150$ & Mesenchymal cells \\
\hline AE1/AE3 cytokeratin & Monoclonal & Pre-diluted by the manufacturer & Epithelial cells \\
\hline Desmin & Monoclonal & $1: 100$ & Leiomyosarcoma \\
\hline $\begin{array}{l}\text { Progesterone receptor (PR) } \\
\text { Oestrogen receptor (OR) }\end{array}$ & Monoclonal & Pre-diluted by the manufacturer & $\begin{array}{c}\text { Well or moderately } \\
\text { differentiated endometrioid cancer }\end{array}$ \\
\hline
\end{tabular}

*All primary antibodies were from DakoCytomation, Poland; * paraffin sections of a gastrointestinal stromal tumour (GIST) positive for CD117 were used as a positive control

contrary, the 'collision hypothesis' assumes that a neoplastic tumour develops from two separate populations of stem cells [3-5].

In the latest edition (2009) of the 'TNM Classification of Malignant Tumours', the same classification was applied for CS as for uterine carcinoma [6]. Based on molecular, epidemiological, genetic and histopathological data, most authors support the monoclonal hypothesis, which states that primary malignant epithelial cells are presumably subjected to mesenchymal metaplasia [3-5], thus, the terms 'sarcomatoid carcinoma of the uterus' or 'metaplastic carcinoma' have been proposed for this neoplasm [3].

The aim of our study was to assess the disease course based on the age of the patient, surgical staging, surgical treatment approach, chemotherapy and radiotherapy. Additionally, we performed histopathological analysis based on haematoxylin and eosin (HE) staining and immunohistochemical staining of histological sections. This assessment included tumour size, stage according to FIGO classification, type of morphological patterns in primary tumours, and metastases. We attempted to separate the predictive factors based on clinicopathologic data and to look into issues surrounding the histogenesis of uterine carcinosarcoma.

\section{Material and methods}

Out of 37 women diagnosed with carcinosarcoma and treated at our hospital, five had the primary tumour in their ovaries. The remaining 32 patients had developed the neoplasm in the uterus. Only uterine carcinosarcoma patients were further analysed. The youngest patient diagnosed with carcinosarcoma of the uterus was 33 years old, and the oldest was 82 . The average age of patients at diagnosis was 65.7 years. In 24 patients, the disease was diagnosed between 2001 and 2006, whereas in eight patients it was diagnosed between 2008 and 2009.
Archival paraffin blocks were sectioned at the Department of Pathomorphology at the Gynaecology and Obstetrics Clinical Hospital in Poznan. Histopathological analysis was based on $\mathrm{H} \& \mathrm{E}$ staining and immunohistochemical staining (Table 1). Immunostaining was performed using the DAKO LSAB + System HRP technique. In brief, epitope retrieval was performed by heating at $95-99^{\circ} \mathrm{C}$ in $\mathrm{pH} 6.1$ modified citrate buffer, Dako Target Retrieval Solution, using a water bath. Next, endogenous peroxidase activity was blocked with $3 \% \mathrm{H}_{2} \mathrm{O}_{2}$ for five minutes. After washing with TBSi buffer solution, the tissue sections were incubated with the primary antibody for 30 minutes and then washed again. Next, the sections were incubated with a biotinylated secondary antibody, from DakoCytomation LSAB+SystemHRP, for 30 minutes and washed. Then, the tissue sections were stained with streptavidin/peroxidase complex, from DakoCytomation LSAB+System-HRP, for 30 minutes and again washed. 3,3'-diaminobenzidine tetrahydrochloride (DAB) solution was applied to tissue sections for ten minutes. Then the tissue sections were again washed, counterstained with haematoxylin, dehydrated and mounted in balsam.

Immunopositive staining observed in at least $10 \%$ of cells was defined as a positive result. The presence of an immunopositive reaction in $10 \%$ of cells was assessed as $1+$; reaction in $50 \%$ of cells was assessed as $2+$; and reaction in more than $60 \%$ of cells was assessed as $3+$. Negative controls were prepared by omitting the primary antibody. Positive controls, for the assessment of CD117 expression, were prepared from tissue sections of gastrointestinal stromal tumour (GIST), which stains positively for CD117.

\section{Results}

\section{Clinical characteristics of the patients}

All 32 patients with a diagnosis of carcinosarcoma of the uterus underwent total hysterectomy with bilateral salpingo-oopherectomy and omentectomy. Only 
one of the patients with stage I advancement of carcinosarcoma also had pelvic lymphadenectomy. Of these, 25 patients were classified as either stage I or II. The remaining seven patients were classified as stage III or IV. In the 25 stage I and II patients, the neoplasms were limited to the uterus. Peritoneal washings obtained from 16 of the 25 were negative for cancer cells. However, in peritoneal washings obtained from the seven patients diagnosed with either stage III or IV, cancer cells were identified. Five of the seven patients had neoplastic infiltration of the ovaries, oviducts, and omentum, while the remaining two also had neoplastic infiltration of the peritoneum and appendix. All patients were subjected to radiation therapy. In addition, patients with cancer stage II, III or IV received a combination chemotherapy regimen. Chemotherapy treatment consisted of platinum, DNA alkylating agents (e.g. ifosfamide), and taxanes that inhibit mitotic activity. Two patients received a combination chemotherapy regimen of CAELYX and CYVADIC. Patients diagnosed with stage III and IV cancer died during the first, second or third year following the initial diagnosis of uterine carcinosarcoma. The deaths were primarily due to either lung metastasis or kidney insufficiency.

\section{Histological structure of uterine carcinosarcomas}

Based on H\&E and immunohistochemical staining, various neoplastic patterns were observed, which are presented in Table 2 .

Among carcinomatous components, endometrioid carcinomas were most abundant: they were observed in $16(50 \%)$ specimens. Clear cell carcinomas were observed in four (13\%) specimens, serous carcinomas were observed in seven $(22 \%)$ specimens, squamous cell carcinomas were observed in two $(6 \%)$ specimens, and undifferentiated were observed in three $(9 \%)$ specimens. We cannot exclude the possibility that squamous patterns originated from squamous metaplasia of endometrioid carcinoma. Most tumour masses (19 of 32) presented mainly a glandular pattern. In the remaining 13, the glandular pattern comprised a smaller portion of the tumour mass. In four of the 13 tumour masses, only single glandular cells were observed. Most of the carcinomas were either poorly (G3) or moderately (G2) differentiated (22). In seven analysed neoplasms, the expression of vimentin was observed in an endometrioid glandular pattern and these cells were also positive for cytokeratin AE1/AE3 (Figures 1,2). Patterns showed progesterone receptor (PR) and oestrogen receptor (OR) expression scored from 0 to $3+$. More differentiated cancers demonstrated PR and OR expression of $2+$
Table 2. Histopathological characteristics of carcinosarcomas

\begin{tabular}{|lc|}
\hline & Number of cases \\
\hline FIGO stage & 16 \\
I & 9 \\
II & 7 \\
III and IV & \\
\hline Types of glandular pattern & 16 \\
Endometrial carcinoma & 4 \\
Clear cell carcinoma & 7 \\
Serous carcinoma & 2 \\
Squamous cell carcinoma & 3 \\
Undifferentiated carcinoma &
\end{tabular}

\begin{tabular}{|c|c|}
\hline Carcinomatous $v$. sarcomatous componen & \\
\hline Carcinomatous patterns predominantly & 19 \\
\hline Sarcomatous patterns predominantly & $\begin{array}{c}13 \text { (4/13 tumours } \\
\text { showed single } \\
\text { distribution of cells) }\end{array}$ \\
\hline
\end{tabular}

$\begin{array}{cc}\text { Grade of cancer differentiation } & \\ 1^{\circ} & 7 \\ 2^{\circ} & 6 \\ 3^{\circ} & 19 \\ \text { Mitotic activity } & >10 / 10 \text { HPF } \\ & \text { (High Power Field) }\end{array}$

\begin{tabular}{|lc|}
\hline Types of sarcomatous pattern & \\
Stromal sarcoma & 12 \\
Leiomyosarcoma & 8 \\
Rhabdomyosarcoma & 2 \\
Chondrosarcoma & 8 \\
Osteosarcoma & 3 \\
Fibrosarcoma & 4 \\
Malignant mesenchymoma & 2 \\
Mitotic activity & $10 / 10 \mathrm{HPF}$ \\
\hline Histological pattern in metastases & \\
Glandular pattern only & 2 \\
Carcinomatous pattern and small & \\
admixture of sarcomatous pattern & 3 \\
predominantly sarcomatous pattern & 2 \\
\hline
\end{tabular}

to $3+$, while poorly differentiated showed $\mathrm{PR}$ and $\mathrm{OR}$ expression of 0 to $1+$. Surprisingly, we did not observe an immunopositive reaction for CD117 in any of the analysed tumours.

The largest group among sarcomatous elements (16) was represented by stromal sarcoma. Most of them (60\%) demonstrated CD10 positive expression. The remaining sarcomas observed, in order of decreasing number, were leiomyosarcoma (desmin positive), chondrosarcoma, fibrosarcoma, osteosarcoma, rhabdomyosarcoma and malignant mesenchymoma. In clinicomorphological analysis, we did not identify the prognostic value of histological structures of carcinosarcomas. 

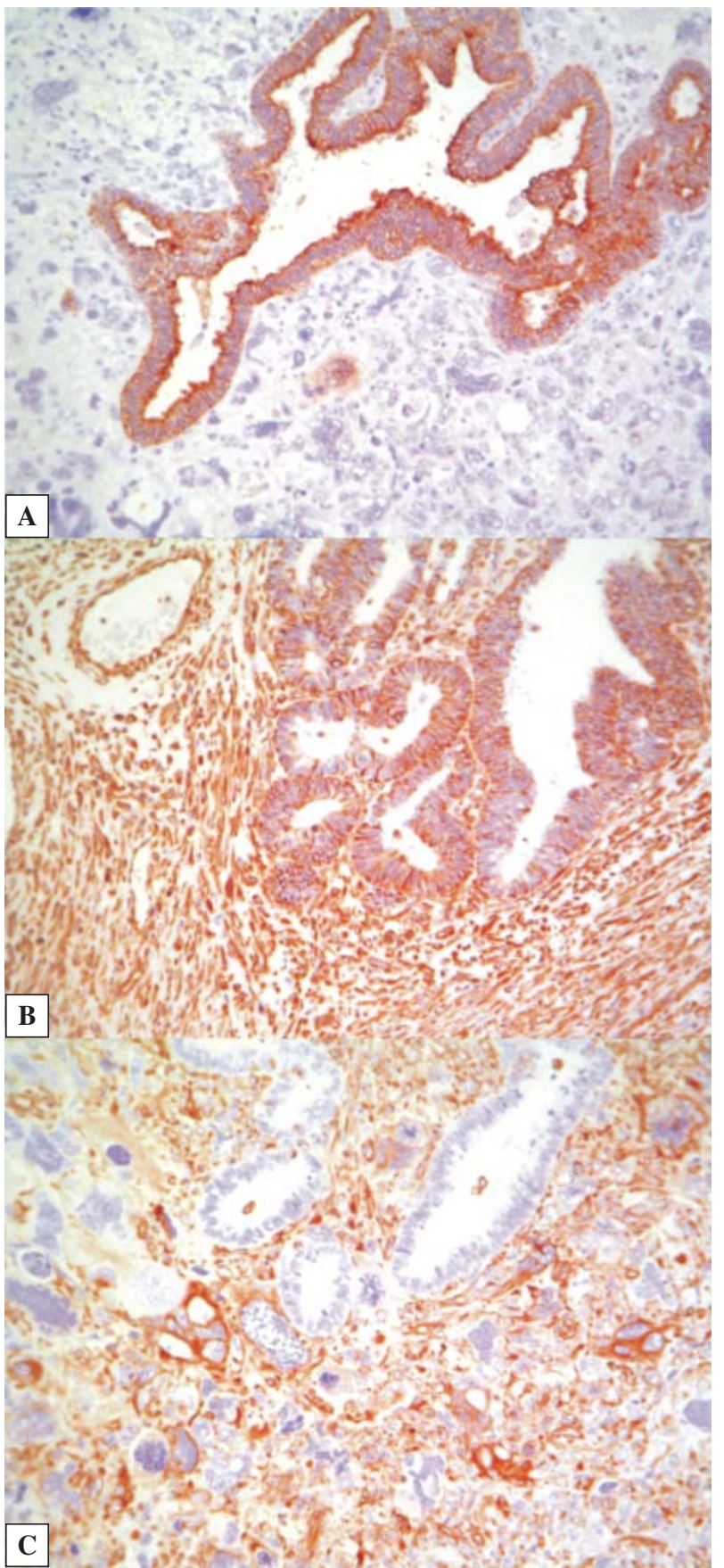

Figure 1. Carcinomatous pattern in carcinosarcoma; immunopositive for cytokeratin (A) and vimentin (B), and immunonegative reaction for vimentin $(\mathbf{C})$. Original magnification $200 \times$

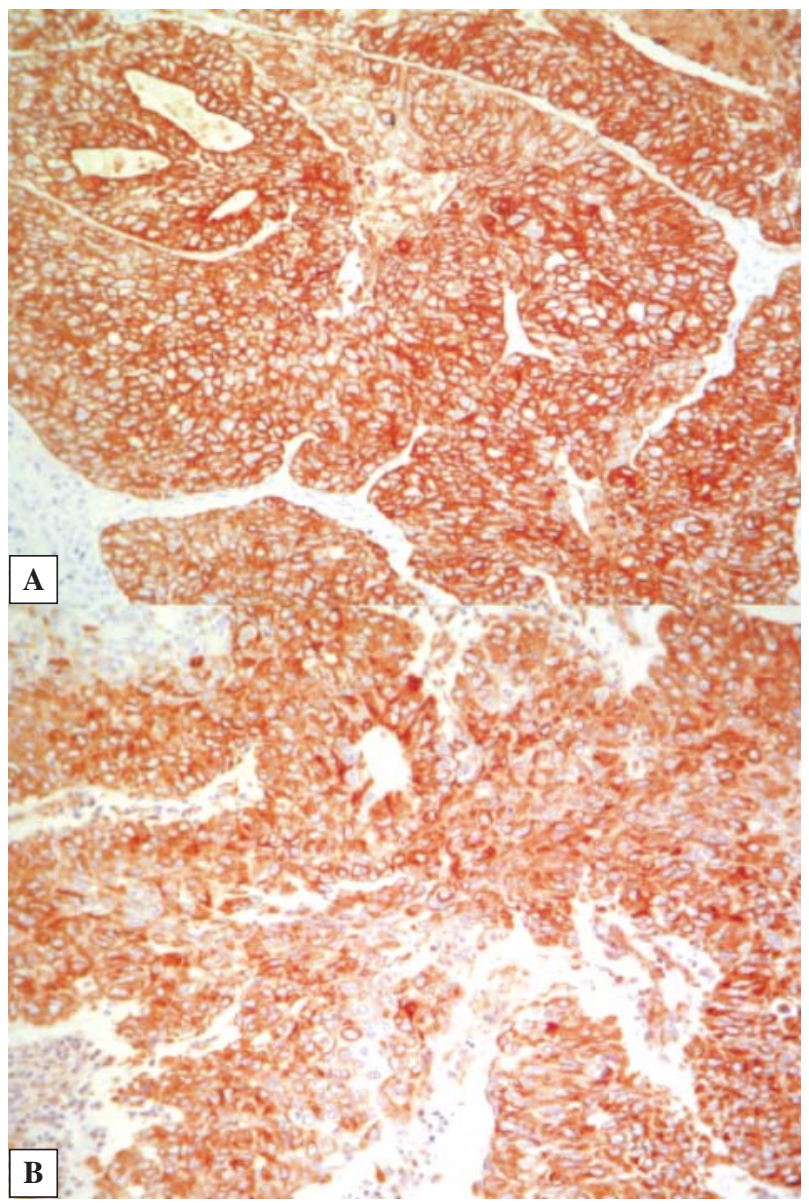

Figure 2. Poorly differentiated carcinosarcoma, immunopositive for cytokeratin (A) and vimentin (B). $200 \times$

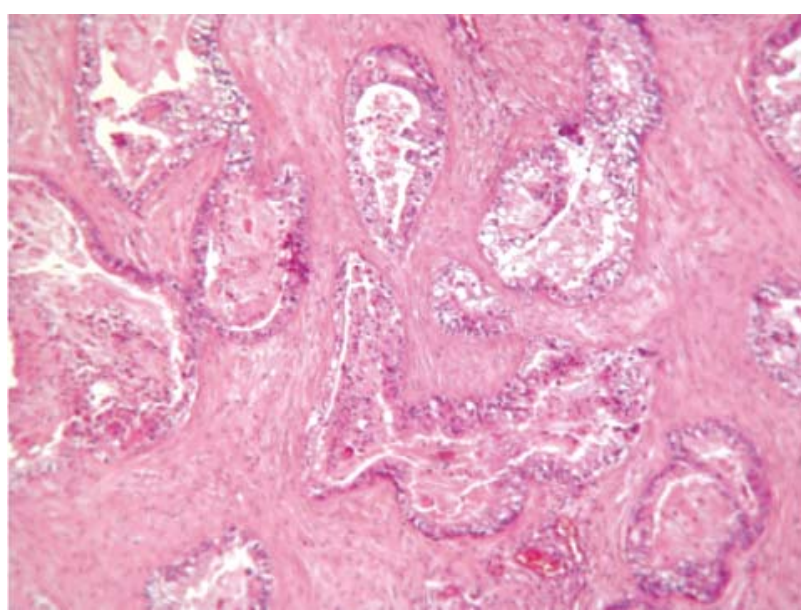

Figure 3. CS metastases to ovary: clear cell pattern. HE staining, $200 \times$

\section{Discussion}

Carcinosarcomas (CS) are tumours where both mesenchymal and epithelial components are of malignant 
nature and are considered one of the most aggressive uterine cancers. Uterine carcinosarcomas are rare. Although, in relation to carcinoma, carcinosarcoma develops in older women, their risk factors are similar. CS incidence increases after pelvic irradiation and exposure to exogenous oestrogens. Since adipose tissue is a source of oestrogen, increased CS incidence may be attributable to obesity [3,9]. Furthermore, tamoxifen administered for breast cancer treatment is associated with a 2-3-fold increase in the risk of CS incidence $[3,8]$.

In carcinosarcomas, among carcinomatous patterns, most commonly listed are endometrioid cancers, while among sarcomatous patterns, commonly observed are stromal sarcomas, and less commonly leiomyosarcomas and chondrosarcomas [3, 4, 11].

Uterine cancer patterns, when considered separately, have different prognoses. Five-year survival for women diagnosed with type I cancer reaches $90 \%$, whereas in those diagnosed with type II cancer, it is between $50 \%$ and $60 \%$, and in those diagnosed with carcinosarcoma it is between $33 \%$ and $39 \%$ [4, 10-13]. Five-year survival for leiomyosarcoma is assessed to be between $15 \%$ and $25 \%$, but for stage I and II it is assessed to be between $40 \%$ and $70 \%$ [14]. Bosquet et al. examined 121 patients with carcinosarcoma of the uterus treated with adjuvant chemotherapy following surgical resection. They found that the fiveyear survival for stage I and II was 59\%, for stage III it was $22 \%$, and for stage IV it was 9\% [4]. Yamade et al. [13] demonstrated five-year survival of $74 \%$ in patients with CS limited to the uterus; however, in the group of patients with CS extension beyond the uterus, five-year survival was only $24 \%$. In our CS series, seven of the 32 patients diagnosed with stage III and IV cancer died during the first, second or third year following the initial diagnosis of uterine carcinosarcoma. According to some authors, radiation therapy does not influence the survival rate of patients diagnosed with carcinosarcoma [13]. Most studies available in the literature are based on small groups of patients, so that conclusions drawn from them may not always be reliable. Comparing the results of treatment with carcinosarcoma morphological patterns, we were unable to find morphological structures which could potentially serve as prognostic markers.

Literature data $[4,15]$ draws attention to the carcinomatous pattern's higher mitotic activity, higher proliferation, and metastatic capabilities compared to the sarcomatous pattern. Our observation was consistent with the studies referenced above $[4,15]$, since we also observed higher mitotic activity of the carcinomatous pattern than of the sarcomatous pattern, and glandular metastases to ovaries in two patients.
Carcinosarcoma metastases to other organs most commonly derive from the carcinomatous pattern [3, $4,15,18]$. It is difficult to explain which factors determine the higher activity of carcinomatous pattern. Several previous genetic studies have emphasised that in both carcinosarcoma pattern types chromosomal aberrations were similar, in that they commonly involved loss of heterozygoticity and mutations in p53, K-ras, C-myc and WT-1 genes [3, 20, 21]. The genetic similarity between the two neoplastic components serves as evidence for the monoclonal origin of carcinosarcoma.

It is worth noting that, in our immunohistochemical studies, cytoplasm of cells in carcinomatous component was positive for vimentin in $22 \%$ of analysed tumours. Concurrently, these cells were also positive for cytokeratin (Figures 1, 2). Based on these results, we speculate that these kinds of cytokeratin-vimentin positive cancer cells may be more easily subjected to metaplasia in spindle-shaped cells forming sarcomatous patterns with simultaneous mutations on different metabolic levels.

Generally, the treatment employed in our centre was consistent with that presented in the literature. First, treatment consisted of total hysterectomy with bilateral salpingo-oophorectomy, omentectomy and peritoneal lavage. The mainstay of treatment is radiation therapy, followed by chemotherapy. Unfortunately, according to the literature, CS are not very sensitive to either chemotherapy or radiation therapy [4, 10, 11]. Numerous studies have recently emerged in which authors have applied Gleevec treatment [16, 17]. Gleevec is a tyrosine kinase inhibitor which inhibits proliferation of neoplastic cells and induces apoptosis [16]. The application of Gleevec is only justifiable in cases of immunopositive reactions of carcinosarcoma to CD117. This cancer drug is well tolerated by patients, but demonstrates minimal activity when used as monotherapy [16]. However, in our series of $32 \mathrm{CS}$ patients, we could not demonstrate in any specimen, in either carcinomatous or sarcomatous pattern, an immunopositive reaction when tested for products of c-kit gene specific for CD117. This contradicts other studies in which immunopositive reactions with CD117 antibodies in CS have been reported $[16,17]$.

The problem of lymphadenectomy in cases of CS of the uterus has not yet been conclusively resolved. It is accepted that while carcinomas migrate mainly via the lymphatic system, most sarcomas spread via the vascular system, predominantly to the abdominal cavity and lungs. In our CS series, only one of the 32 patients had pelvic lymphadenectomy. Randomised studies of endometrial cancers have neither demon- 
strated longer survival rates nor later metastases in women who underwent lymphadenectomy compared to those who did not $[22,23]$. In patients with uterine wall infiltration greater than $1 / 2$ (Ib stage), Park et al. found that $32 \%$ of patients had metastases in lymph nodes [19], while Bitterman et al. found metastases mainly in lymphatic ducts of ovaries, fallopian tubes and omentum [15]. The authors of these studies do not recommend routine lymphadenectomy as part of endometrial cancer treatment. However, the benefits of lymphadenectomy in the treatment of CS are still open to discussion.

In conclusion, cytokeratin-vimentin positive cells in adenocarcinoma suggest either the possibility of sarcomatous metaplasia of adenocarcinoma, or a mesenchymal component arising from stem cell transformation giving rise to different cell lines. In this study, we did not find a prognostic value of histological structures of carcinosarcomas of the uterus.

\section{References}

1. Brooks SE, Zhan M, Cote T, Baquet CR. Surveillance, epidemiology, and end results analysis of 2677 cases of uterine sarcoma 1989-1999. Gynecol Oncol. 2004;93:204-208.

2. Vaidya AP, Horowitz NS, Oliva E, Halpern EF, Duska LR. Uterine malignant mixed mullerian tumors should not be included in studies of endometrial carcinoma. Gynecol Oncol. 2006;103:684-687.

3. Kernochan LE, Garcia RL. Carcinosarcomas (malignant mixed Mullerian turmor) of the uterus: advances in elucidation of biologic and clinical characteristics. J Natl Compr Canc Netw. 2009;7:550-556.

4. Gonzalez Bosquet J, Terstriep SA, Cliby WA et al. The impact of multi-modal therapy on survival for uterine carcinosarcomas. Gynecol Oncol. 2010;116:419-423.

5. Gorai I, Yanagibashi T, Taki A et al. Uterine carcinosarcoma is derived from a single stem cell: an in vitro study. Int J Cancer. 1997;72:821-827.

6. Sobin LH, Gospodarowicz MK and Wittekind Ch eds. International Union Against Cancer (UICC). TNM Classification of Malignant Tumours. 7th ed. Hoboken, NJ: Wiley-Blackwell; 2009:1-310.

7. Zelmanowicz A, Hildesheim A, Sherman ME et al. Evidence for a common etiology for endometrial carcinomas and malignant mixed mullerian tumors. Gynecol Oncol. 1998;69:253-257.

8. Curtis RE, Freedman DM, Sherman ME, Fraumeni JF Jr. Risk of malignant mixed mullerian tumors after tamoxifen therapy for breast cancer. J Natl Cancer Inst. 2004;96:70-74.

9. Sebenik M, Yan Z, Khalbuss WE, Mittal K. Malignant mixed müllerian tumor of the vagina: case report with review of the literature, immunohistochemical study, and evaluation for human papilloma virus. Hum Pathol. 2007;38:1282-1288.
10. Schweizer W, Demopoulos R, Beller U, Dubin N. Prognostic factors for malignant mixed müllerian tumors of the uterus. Int J Gynecol Pathol. 1990;9:129-136.

11. Nielsen SN, Podratz KC, Scheithauer BW, O?Brien PC. Clinicopathologic analysis of uterine malignant mixed müllerian tumors. Gynecol Oncol. 1989;34:372-378.

12. Kędzia H, Kędzia W eds. Neoplasms of female genital tract. Pathomorphologic diagnosis. Clinical proceedings. Wrocław, Poland: MedPharm; 2010:1-282.

13. Yamada SD, Burger RA, Brewster WR, Anton D, Kohler MF, Monk BJ. Pathologic variables and adjuvant therapy as predictors of recurrence and survival for patients with surgically evaluated carcinosarcoma of the uterus. Cancer. 2000;88: 2782-2786.

14. Abeler VM, Royne O, Thoresen S, Danielsen HE, Nesland JM, Kristensen GB. Uterine sarcomas in Norway. A histopathological and prognostic survey of a total population from 1970 to 2000 including 419 patients. Histopathology. 2009; 54:355-364.

15. Bitterman P, Chun B, Kurman RJ. The significance of epithelial differentiation in mixed mesodermal tumors of the uterus. A clinicopathologic and immunohistochemical study. Am. J Surg Pathol. 1990;14:317-328.

16. Huh WK, Sill MW, Darcy KM et al. Efficacy and safety of imatinib mesylate (Gleevec) and immunohistochemical expression of c-Kit and PDGFR-beta in a Gynecologic Oncology Group Phase II Trial in women with recurrent or persistent carcinosarcomas of the uterus. Gynecol Oncol. 2010; 117:248-254.

17. Leath CA $3^{\text {rd }}$, Straughn JM Jr, Conner MG et al. Immunohistochemical evaluation of the c-kit proto-oncogene in sarcomas of the uterus: a case series. J Reprod Med. 2004; 49:71-75.

18. Schipf A, Mayr D, Kirchner T, Diebold J. Molecular genetic aberrations of ovarian and uterine carcinosarcomas - a CGH and FISH study. Virchows Arch. 2008;452:259-268.

19. Park JY, Kim DY, Kim JH, Kim YM, Kim YT, Nam JH. The role of pelvic and/or para-aortic lymphadenectomy in surgical management of apparently early carcinosarcoma of uterus. Ann Surg Oncol. 2010;17:861-868.

20. Abeln EC, Smit VT, Wessels JW, de Leeuw WJ, Cornelisse CJ, Fleuren GJ. Molecular genetic evidence for the conversion hypothesis of the origin of malignant mixed müllerian tumours. J Pathol. 1997;183:424-431.

21. Schulten HJ, Gunawan B, Enders C, Donhuijsen K, Emons $\mathrm{G}$, Füzesi L. Overrepresentation of $8 \mathrm{q}$ in carcinosarcomas and endometrial adenocarcinomas. Am J Clin Pathol. 2004;122:546-551.

22. ASTEC study group, Kitchener H, Swart AM, Qian Q, Amos C, Parmar MK. Efficacy of systematic pelvic lymphadenectomy in endometrial cancer (MRC ASTEC trial): a randomised study. Lancet. 2009;373:125-136.

23. Panici PB, Basile S, Maneschi F et al. Systematic pelvic lymphadenectomy vs. no lymphadenectomy in early-stage endometrial carcinoma: randomized clinical trial.J Natl Cancer Inst. 2008;100:1707-1716.

Submitted: 1 October, 2012

Accepted after reviews: 14 November, 2012 